\title{
Preparation and Characterization of Intramuscular PLGA Based Microsphere
}

\author{
Prateek Pathak and Dr. Sarvesh Paliwal \\ Department of Pharmacy, Banasthali Vidyapith, Rajasthan, India
}

\begin{abstract}
The current studies was design and evaluate biodegradable PLGA microspheres for sustained or extended release, with primary goal of avoiding combination of oral therapy for the treatment of schizophrenia. PLGA copolymers 75:25 was used to prepare four microsphere formulations of anti-psychotic drug Olanzapine. The microspheres were characterized by in-vitro dissolution and other physio-chemical methods. A simulation of multiple dosing at weekly or 15-day regimen revealed pulsatile behavior for all formulations with steady state may be achieved by the second dose. Overall, the in-vitro study of Formulations 001, 002, 003, or 004 will eliminate the need for combination oral therapy and reduce time to achieve steady state, with a smaller washout period upon duration of therapy. Results of this study prove the suitability of using PLGA 75:25 copolymers of different composition and molecular weight to produce sustained or extended release formulations that can enhance pharmacological effectiveness for anti-psychotic intra-muscular administration of Olanzapine.
\end{abstract}

Keywords: sustained release, PLGA microspheres, Olanzapine

Article Info: Received 06 July 2019; Review Completed 12 August 2019; Accepted 19 August 2019; Available online 15 Sep 2019

\section{Cite this article as:}

Pathak P, Paliwal S, Preparation and Characterization of Intramuscular PLGA Based Microsphere, Journal of Drug Delivery and Therapeutics. 2019; 9(5):127-130 http://dx.doi.org/10.22270/jddt.v9i5.3631

*Address for Correspondence:

Prateek Pathak, Department of Pharmacy, Banasthali Vidyapith, Rajasthan, India

\section{INTRODUCTION}

Antipsychotic agents involve a variety of compounds that are useful in the treatment of psychosis including schizophrenia. Antipsychotics were previously known as neuroleptics as these agents have ability to reduce the response to emotional and physical stimulation in disturbed or psychotic patients without any effect on consciousness.(1)

The term psychosis means mental disorders indicated by symptoms like reduced and fragmental ability to understand information and reach to logical conclusion, catatonic or disorganized behavior, hallucination, delusion, excitement or violence.(2)

Lifelong psychiatric disorder may be characterized as schizophrenia which is primarily associated with delusional, illogical and paranoid thoughts. Schizophrenia normally may have its onset in early adulthood with remission and exacerbations may appear throughout the life. It is estimated that about $1 \%$ population of world or India is affected by schizophrenia.(3) Symptoms of schizophrenia start with highly diverse set of clinical conditions in late adolescence or early adulthood. Schizophrenic symptoms are dividend in positive and negative sign or symptoms. Positive signs are the first to attract attention to the disorder; these include auditory hallucination normally where patients thought are perceived to be receiving from others or some sources like radio or cell phones. Disorganized speech, repetitive behavior delusions are the other positive sign in schizophrenia. Negative sign or symptoms are less seen but more sustainable and disturbing. These include social withdrawal, anhedonia, alogia, avolition, disorganized thought with blunted affects. Reduced cognitive functions like loss of memory and attention defects may also be there.(4)

Microspheres have now been considered as reliable and effective release system which reduces dose, dosing frequency, and possibility of dose dumping with high patient compliance (5). Microspheres prepared by innate or artificial polymers provides sustained release, for instance polyesters used in microspheres, have the benefit of using water as a vehicle to form suspensions which offers constant release of desired drug. It has been documented that release of a drug from microsphere is directly influenced with certain factors viz. physicochemical properties of a drug, primary burst and excipients (6).

Injectable microsphere systems were developed in late 70's. Poly (lactide) and/or poly (lactide-co-glycolide)(7-10)was used to encapsulate an ester of norethisteron called NET via 
oil-in-water emulsion/solvent evaporation process. These microcrystals of NET were encapsulated into microspheres of PLA and PLGA. Since then PLGA has been the first choice of polymer for preparation of microspheres to encapsulate APIs using $\mathrm{w} / \mathrm{o}$ or $\mathrm{o} / \mathrm{w}$ emulsion/solvent evaporation technique(6-7). Poly ( $\varepsilon$-caprolactone) is the secondly most accepted polymer for preparation of microspheres to encapsulate APIs. Moreover PLGA, PCL and PLA, diblock copolymers of lactide, ethylene glycol, L-lactide and $\varepsilon$ caprolactone, triblock co polymers of caprolactons, lactides and glycolides, have also been used to encapsulate APIs via o/w emulsion / solvent evaporation. (6)

Biodegradation rate of the microspheres is directly linked to its composition which in turn affects the release profiles of API. For instance as PGA, PLA and PCL have hydrolytic degradation rate in order of PGA > PLA >> PCL. PCL have excellent permeability in case of steroidal drugs but it shows slow biodegradation, in contrast, PLA and PGA had inferior permeability in case of steroidal drugs but it shows homogenous biodegradation. It has been observed that microspheres of PLGA get degraded quicker and release of API is more rapid from the microspheres when hydrophilic glycolide component is increased in the system. The polymer's chirality, density of cross linking and loading of drugs in microspheres also influences the release profiles of API. Casein and chitosan microspheres shows slower release of API particularly when the amount of cross linking is increases significantly as it results in increased barrier density for drug diffusion, with reduction in the drug loading. Higher initial burst in microspheres has been observed when un-encapsulated drug is available in higher quantity on the surface of microspheres.(6)

Olanzapine: (11-12)



IUPAC Name: 2-methyl-4(-4-methyl-l-piperazinyl)-10Hthieno-[2,3-b][1,5]-benzo-di- azepine.

$\begin{array}{ll}\text { Molecular formula: } & \mathrm{C}_{17} \mathrm{H}_{20} \mathrm{~N}_{4} \mathrm{~S} \\ \text { Molecular Weight: } & 312.4\end{array}$

Solubility: water insoluble, in moderation soluble in acetonitrile, soluble in organic solvents such as DMSO and dimethyl formamide.

Category: Antipsychotic.

Description: A yellow crystalline powder.

\section{MATERIALS AND METHODS}

\section{Materials}

Olanzapine was obtained as a gift sample from Jubilant Pharmaceutcals, India, and PLGA 75:25 from Resomer provided by Amneal Pharmaceuticals, India. All other chemicals were also obtained commercially as Pharmaceutical grade reagents by Amneal Pharmaceuticals, India.

\section{Preparation of Microspheres}

Several methods for preparation of microsphere are reported in literature. In some cases, slight variations in basic methods are also reported. These variations in basic methods can produce numerous changes in final outcome. However, some of the basic techniques for microsphere preparation were gathered from literature survey. Interfacial polymerization technique is normally used for encapsulation of liquid. This technique involves two monomers one is water soluble and other is oil soluble. However, this process few limitations like reaction between active substance and reagent, toxicity produced due to untreated monomer, degradation resulted by monomer reaction and high permeability of coating and fragility of microsphere.

Another method is water immiscible liquid core in which isocynate or some other polymers are used. Various modification of coacervation phase separation techniques are used however in present study solvent extraction method was used for preparation of long acting intra muscular microsphere. This technique is simple with high yielding and economic. It has been found that this method gives reproducible results. Equipments required in this technique were available in the laboratory i.e. Static mixer peristaltic pump, tanks of different capacity sieve of different pore size.

\section{Method of Preparation}

In the present study solvent extraction method has been used. A static mixture was used to reduce the globule size of primary emulsion prepared passing through the static mixer. Two separate phases one dispersing phase containing polymer and API in organic solvent(s) was mixed or homogenized with dispersion media, containing aqueous solution of PVA and ethyl acetate at a predefined rate. The primary emulsion was mixed with quenching liquid with lesser amount ethyl acetate in water at $6-7^{\circ} \mathrm{C}$ for 4 hours with constant stirring.

This process was used for slow solvent extraction from microspheres. The microspheres after solidification were passed through the sieve to remove particles of less than 25 microns and more than 500 microns. The collected microspheres were lyophilized at $5^{\circ} \mathrm{C}$ for 48 hours. This method was used for preparation of placebo microspheres, however for microspheres containing API; was dissolved in benzyl alcohol and mixed with polymer solution to achieve disperse phase. The microspheres were characterized as described in Section Characterization of Microspheres.

\section{Characterization of Microspheres}

\section{Particle Size}

Particle sized measurement of prepared formulation was done by Bacman coulter counter. Sample was prepared by suspending microspheres $1 \mathrm{mg}$ per $\mathrm{mL}$ in distilled water along with $0.1 \% \mathrm{w} / \mathrm{v}$ polysorbate 80 as surfactant. Readings for D10, D50 and D90 were recorded for each sample. Each sample was run in triplicate and mean value were taken for study.

\section{Surface Morphology}

The surface morphology was examined by scanning electron microscopy (SEM) (Jeol JSM 17100 JEC-300 FC Auto fin coater) at an appropriate magnification, after palladium/gold coating of the microsphere sample on an aluminum stub. 


\section{Drug Content}

Olanzapine content in the microspheres was analyzed by HPLC method using a Column C18, Drug content (\%) was expressed as the "weight of drug in microspheres/weight of microspheres $\times 100$."

\section{RESULTS AND DISCUSSION}

\section{Particle Size Measurement of Microsphere}

Particle size measurement was determined with the help of laser diffraction method for all four formulations. Results are presented in Table 1, D90 of all formulations was found in good agreement with previous studies. Similarly, D50 values are also acceptable for all four formulations.

Table 1 : Particle Size results of finished formulation

\begin{tabular}{|c|c|c|c|c|}
\hline Sr. No. & Formulation Code & $\begin{array}{c}\text { Mean Value } \\
\text { D10 }\end{array}$ & $\begin{array}{c}\text { Mean Value } \\
\text { D50 }\end{array}$ & $\begin{array}{c}\text { Mean Value } \\
\text { D90 }\end{array}$ \\
\hline 1 & Formulation 400-PP001 & 49.4 & 89.6 & 148.3 \\
\hline 2 & Formulation 400-PP002 & 41.8 & 88.7 & 154.0 \\
\hline 3 & Formulation 400-PP003 & 46.5 & 86.7 & 158.4 \\
\hline 4 & Formulation 400-PP004 & 41.5 & 83.9 & 145.9 \\
\hline
\end{tabular}

\section{Surface Morphology of Microspheres}

The SEM images presented for microspheres were observed at magnification X60 and X2000. The images revealed that spherical shape of drug particles in developed formulation in comparison to microspheres where particles were found segregated with smooth surface without crack and pores. The images are shown in figure 1.



Figure 1: image at $5.0 \mathrm{kV}$ and $\mathrm{x} 60$

\section{Drug Content of Microsphere}

For Drug content of olanzapine in final product (long acting microspheres) and to determine the percent drug loading of olanzapine, HPLC method with UV detector was used. Standard solution of olanzapine was used as reference and peak area of standard was used to calculate the quantities of olanzapine in sample solution. Similar process was used assayed for four selected batches. Assay of all four formulations was found satisfactory in terms of drug loading. Results are mentioned in Table:2

Table 2: Assay of olanzapine finished formulation

\begin{tabular}{|c|c|c|c|}
\hline Sr.No: & Formulation Code & Assay & \% Drug Loading \\
\hline 1 & Formulation 400-PP001 & 96.25 & 38.5 \\
\hline 2 & Formulation 400-PP002 & 97.5 & 39.0 \\
\hline 3 & Formulation 400-PP003 & 94.75 & 37.9 \\
\hline 4 & Formulation 400-PP004 & 98.75 & 39.5 \\
\hline
\end{tabular}




\section{CONCLUSIONS}

From the present study, it is concluded that solvent evaporation method can be used successfully for preparation of microspheres. The size of the microspheres can be controlled well with an ideal polymer used in the formulations.

Intramuscular depot forming microspheres are widely used for extended release under special conditions like psychosis, schizophrenia and related conditions where frequent dosing is not possible due to patient non compliance. Present work is done on olznzapine which is antipsychotic in action. In vivo release rate studies of the formulation is still due. However in vitro release rate study at elevated temperature is found to be satisfactory. If biological studies (Release rate, bioavailability and duration of action) are found satisfactory the process may be used for commercial production of formulation.

Finally, it can be calculated that Olanzapine microspheres may be formulated using said process and parameters with desire sustain action.

\section{ACKNOWLEDGMENTS}

Authors are thankful to Department of Pharmacy Banasthali Vidyapith Jaipur, Rajasthan for providing support while carrying out this research work. One of the authors Prateek Pathak is thankful and obliged to Amneal Pharmaceuticals Ahmadabad Gujarat for providing all logistic support and permission to carry out this research work. He is also thankful to Jubilant Pharmaceuticals, India, for providing gift sample of Olanzapine.

\section{REFERENCES}

1. Limbird L.E. and Hardmen J.G. Goodmen \& Gilman's The Pharmacological basis of Therapeutics, $10^{\text {th }}$ Edition, page 485 514.

2. Kane J, M., "Pharmacologic treatment of schizophrenia," Biological Psychiatry, 1999; 46(10):13961408.

3. Johnson D, "Long-term drug treatment of psychosis: observations on some current issues," International Review of Psychiatry, 1990; 2:341-353.

4. Wyat R. J., "Neuroleptics and the natural course of schizophrenia," Schizophrenia Bulletin, 1991; 17(2):325-351.

5. D’Souza, S.S. and Deluca, P.P., "Development of a Dialysis In vitro Release Method for Biodegradable Microspheres", AAPS Pharmaceutical Science and Technology, 2005; 6(2):42.

6. Wu, Linfeng, "Long Acting Injectable Hormonal Dosage Forms for Contraception", Pharmaceutical Research, 2015; 32(2):180-219.

7. D'Souza S.S. and Deluca P.P., “A Short term Quality Control Tool for Biodegradable Microspheres", AAPS Pharmaceutical Science and Technology, 2014; 15(3):530541.

8. Petrova, E. A., "Influence of Microencapsulation Process Parameter on Naltrexone Prolnged Release Dosage Form", Pharmaceutical Chemistry Journal, 2014; 48(1)65-68.

9. D'Souza S., "IVIVC from Long Acting Olanzapine Microspheres", International Journal of Biomaterials Volume 2014 (2014), Article ID 407065, Page 11.

10. D'Souza , S., " Preparation, Characterization and In Vivo Evaluation of Olanzapine Poly (D,L-lactide-co-glycolide) Microspheres", Journal of Pharmaceutics, Volume 2013(2013), Article ID 831381, Page 9.

11. Ministry of Health and Welfare, Government of India, “Indian Pharmacopoeia 2010", The Indian Pharmacopoeia Commission, Ghaziabaad, volume III, 2010, Page 1811.

12. Sweetman, Sean C., "Martindale: The Complete Drug Reference", Pharmaceutical Press, 36th Edition, 2009, Page 1012-1014. 